\title{
Expression of cocaine- and amphetamine-regulated transcript in the pancreas of the European bison
}

\author{
SYLWIA MOZEL, MAŁGORZATA MATYSEK, ANNA ZACHARKO-SIEMBIDA, \\ RADOS $Ł A W$ SZALAK, MICHAŁ KRZYSIAK*, **, MARCIN B. ARCISZEWSKI \\ Department of Animal Anatomy and Histology, Faculty of Veterinary Medicine, \\ University of Life Sciences in Lublin, Akademicka 13, 20-950, Lublin, Poland \\ *Białowieża National Park, Park Pałacowy 11, 17-230 Białowieża, Poland \\ **Department of Food Hygiene and Public Health Protection, \\ Warsaw University of Life Sciences - SGGW, Nowoursynowska 159, 02-776 Warszawa, Poland
}

Mozel S., Matysek M., Zacharko-Siembida A., Szalak R., Krzysiak M., Arciszewski M. B. Expression of cocaine- and amphetamine-regulated transcript in the pancreas of the European bison

Summary

Cocaine- and amphetamine-regulated transcript (CART) is a recently discovered neuropeptide thought to mainly act in most laboratory mammals and humans as anorexigenic factor. The expression of CART in wild living animals is barely known. In the present study immunohistochemical stainings were applied to identify CART-immunoreactive (IR) structures in the pancreas of European bison. Antibodies against neuronal marker $\mathrm{Hu} C / \mathrm{D}$ were used to visualize intrapancreatic neurons. The expression of CART was detected in approx. $75 \%$ of $\mathrm{Hu}$ C/D-IR intrapancreatic neurons which may thus also act as interneurons. Additionally, in most intrapancreatic ganglia single CART-IR non-varicose nerve fibers running between neurons were found. Pancreatic blood vessels as well as intralobular ducts were sparsely innervated with CART-IR nerve fibers. Moderately numerous CART-IR nerve terminals were found to innervate the pancreatic endocrine and exocrine tissue. None of islet endocrine cells showed the expression of CART. No presence of CART-IR neuronal elements were found in external connective tissue capsule and septa penetrating inside to the organ. Our study is the first to outline the presence of some differences in CART-ergic innervation pattern of the pancreas between domestic and wild mammals. The lack of CART-IR endocrine islet cells in the pancreas of European bison is an interesting finding, nevertheless its significance is largely unknown at the moment and needs to be further investigated.

Keywords: CART, neuropeptides, immunofluorescence, wild species

Over the last decades the huge efforts have been made to explain and understand the mechanisms of neuronal regulation of the mammalian pancreas. In general it is well known that both the exocrine and endocrine pancreas are controlled by neurons located in different ganglia including those of the autonomic nervous system (15), intram ural ganglia of the enteric nervous system (ENS) (16) and intrapancreatic ganglia (8). Using various research methods it has been demonstrated that some differences in extrinsic and intrinsic innervation patterns of the pancreas exist between various animal species. For example, in humans the exocrine pancreas is richly innervated by parasympathetic and sympathetic nerve fibers whereas in the mouse the autonomic nerve fibers are incidentally found (6). A bulk of biochemically active peptides and enzymes have been recognized as potentially involved in pancreatic juice and hormones secretion but so far only some of them have been found to be of functional importance $(1,4,10,17)$. Cocaine- and amphetamine-regulated transcript (CART) is one of the neuropeptides belonging to a wide group of signaling molecules (18) that predominantly are widely expressed in the central nervous system (7). However in the last years, immunoreactivity to CART has been also found at the periphery, mostly in enteric neurons (3), but also in sensory and parasympathetic neurons of the head (12) or even in intrapancreatic neurons (3).

So far, peripheral regulative role(s) of CART are rather poorly understood and this neuropeptide still attracts the attention of relatively few researchers. A review of the world literature clearly indicates that the studies concerning the intrinsic and extrinsic innervation of the pancreas of the wild living animal species 
are very limited. Therefore the aim of the present study was to immunohistochemically study the expression patterns of CART in the pancreas of the European bison. The choice of the European bison is justified since in recent years huge efforts have been made to restitute this animal species, and nowadays bisons suffer from many undefined conditions, including alimentary tract disorders. The obtained results may also serve as an intriguing starting point for comparative analysis.

\section{Material and methods}

Animals, tissue sampling and processing. The study material was obtained from four $(\mathrm{n}=4)$ adult male European bisons from Białowieża Forest (Poland) that were released from captivity as subadults and lived in wilderness conditions. Because of the presence of necrotic disease of the external genital organs (balanoposthitis), Białowieża National Park (BNP) had the decision of polish authorities of environmental protection to eliminate the sick animals. The permission for tissue sampling was regulated by Polish law. After the death of the animals the abdomen was opened and the whole pancreas were localized, dissected out and fixed in Stefanini solution (paraformaldehyde with picric acid) for 3 days. Next, specimens of the pancreas were cryoprotected in $16 \%$ sucrose solution with addition of sodium azide. Afterwards, the tissues were frozen and cut using a cryostat into $10 \mu \mathrm{m}$ sections. Every tenth section was mounted on chrome alum gelatin-coated glass slides and stored in $-70^{\circ} \mathrm{C}$ for further immunohistochemical analyses.

Immunohistochemistry. The cryostat sections were incubated at room temperature (RT) in $0.01 \mathrm{M}$ phosphatebuffered saline (PBS) with 10\% normal goat serum, $0.25 \%$ Triton X-100 and $0.25 \%$ bovine serum albumin (SigmaAldrich) to inhibit non-specific background staining $(3 \times$ $15 \mathrm{~min}$ ). Next, the sections were incubated overnight in a humid chamber (RT) with a mixture of the following primary antibodies: rabbit anti-CART $(1: 5000$, Phoenix Pharmaceuticals, Burlingame, CA, USA; code H-003-61) and mouse anti-Hu C/D (1:800, Molecular Probes, Eugene, OR, USA; code A-21271). Hu C/D antibody was used as pan-neuronal marker. Thereafter, the surplus of primary antibodies was removed from sections and the samples were washed in PBS $(3 \times 15 \mathrm{~min})$. In order to detect antigen-antibody bindings the slides were incubated $(1 \mathrm{H}, \mathrm{RT})$ in a mixture of species specific secondary antibodies: Texas Red-conjugated rabbit IgG (1 : 400, MP Biomedicals, Cleveland, OH, USA; code 55675) and FITC-conjugated mouse IgG ( $1: 300$, MP Biomedicals, Cleveland, OH, USA; code 55493) were used. After the incubation the excess of secondary antisera was cleared away and slides were once again washed with PBS $(3 \times 15 \mathrm{~min})$. Finally, the slides were mounted in phosphate buffered glycerol $(\mathrm{pH}=8.2)$. Negative control stainings were carried out by using nonimmunoreactive sera instead of primary antibodies and by the application of serum preabsorbed with an antigen excess. In the control sections, no specific immunoreactions were observed.

Image analysis and quantification. The sections were viewed under a spinning disc confocal microscope (BX-DSU Olympus, Nagano, Japan), equipped with appropriate filters to detect fluorochromes: Texas Red (545-580 nm; MWIY2), FITC (470-490 nm; MNIBA2). All images were acquired by digital color camera (DP-70, Olympus). The density of CART-IR nerve fibers in pancreases of the European bisons was estimated visually in accordance with the following semi-quantitative scale: very numerous $(++++)$, numerous $(+++)$, moderate $(++)$, single $(+)$, absent $(-)$. Only neurons with clearly apparent neurons were counted. The prevalence of CART-IR positive intrapancreatic neurons was expressed as a percentage of the total number of $\mathrm{Hu}$ C/D-positive neurons. All numerical data are presented as means \pm SEM. Statistical analysis was made by one-way of variance test ANOVA.

\section{Results and discussion}

In order to evaluate the presence of CART in intrapancreatic neurons we used pan-neuronal marker $\mathrm{Hu}$ $\mathrm{C} / \mathrm{D}$. Neuronal perikarya stained with $\mathrm{Hu} \mathrm{C} / \mathrm{D}$ were bright and easily distinguished from the background. The use of rabbit anti-CART antibodies resulted in stained structures found in distinct regions of the pancreas of the European bison. In the vast majority of intrapancreatic neurons $(75.5 \pm 5.4 \%)$ the expression of CART was found (Fig. 1A). The population of CART-expressing intrapancreatic neurons was statistically higher in relation to CART-negative perikarya $(\mathrm{P}<0.05)$. In some intrapancreatic neurons the immunoreactivity to CART was visualized as granules scattered throughout the neuronal cell body whereas in others CART was ubiquitously present in the whole neuronal soma (except nucleus). In the intrapancreatic ganglia, CART-IR nerve fibers close to CART-IR neurons and CART-negative neurons were incidentally found. Single to moderate numbers of CART-IR nerve fibers were found to innervate the pancreatic acini (Fig. 1A). Moderate numbers of CART-expressing nerve fibers frequently encircled the pancreatic islets but rather incidentally penetrated inside the islet (Fig. 1B). No presence of CART-expressing islet endocrine cells was found. Single (non-varicose) CARTpositive nerve fibers were found in close vicinity to pancreatic ducts and blood vessels (both small veins and arterioles; Fig. 1C). Neither pancreatic external capsule nor septa-forming connective tissue contained CART-expressing nerve terminals.

The results obtained in the study clearly demonstrated that CART is present in both neuronal cell bodies and nerve fibers of the pancreas of the European bison; however, to a varying degree. The moderate numbers of CART-nerve fibers were found around islets and in acini, whereas single CART-expressing nerve fibers were detected between intrapancreatic neurons, in close vicinity to ducts and blood vessels. Because we also found a numerous population of (approx. 75\%) CART-IR intrapancreatic neurons it is likely that the observed CART-IR nerve fibers supplying the pancreas of the European bison are intrinsic and originate in intrapancreatic ganglia. Such a manner of rich CART- 

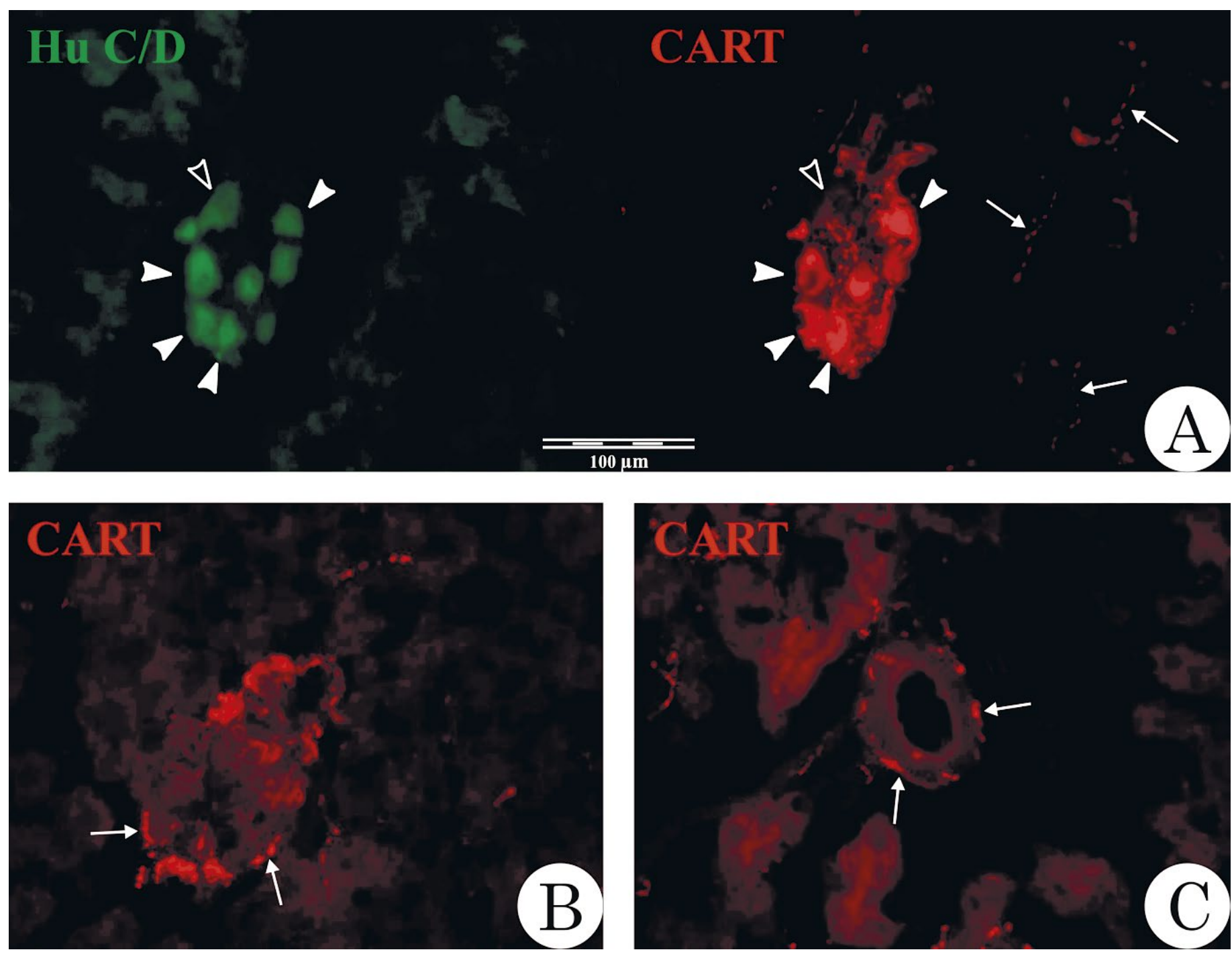

Fig. 1. Expression of CART in frozen sections of the pancreas of the European bison. In Fig. 1A the expression of CART in $\mathrm{Hu}$ C/D-positive intrapancreatic neurons (arrowheads) is seen. Note the presence of Hu C/D-immunoreactive (IR) neurons lacking CART (hollow arrowhead). In Fig. 1B the expression of CART in nerve fibers (arrows) supplying the pancreatic islets is presented. The innervation of pancreatic blood vessels by CART-IR nerve fibres (arrow) is illustrated in Fig. 1C

ergic innervation was also observed in the pancreas of other mammals. High proportions of CART-expressing intrapancreatic neurons were also found in the pancreas of sheep (3), cows (12), pigs (20) and humans (13). We also found CART-IR nerve fibers lying in close vicinity to pancreatic blood vessels which is in line with previous reports. However, to our surprise we found some discrepancies in the numbers of CART-IR nerve fibers supplying the particular regions of the pancreas. Namely, we found that the numbers of CART-IR nerve fibers supplying the exocrine pancreas of the European bison are comparable to that previously described only in the cow (12). The exocrine pancreas of pigs (20), sheep (3) and humans (13) was richly innervated by CART-IR nerve fibers. It is postulated that CART in the mammalian pancreas may exert a stimulatory effect on the exocrine secretion since intravenous infusion of CART substantially increased amylase secretion (5). In the present study we found no presence of CART in nerve fibers supplying the pancreatic parenchyma which remains in contrast to what was previously obtained from sheep (3). Moreover, in the European bison none of the endocrine cells showed the expression of CART which is in line with previous reports obtained from pigs (20). Nevertheless, the presence of single CART-containing endocrine cells that were found either in the islet or embedded throughout the exocrine tissue was reported in sheep (3), cows (12), African ice rats (9), rats (14) and humans (13). In a series of functional studies it has been shown that in pancreatic islet CART-IR endocrine cells the presence of somatostatin and peptide YY can be found (9), which leads to the conclusion that CART is present in delta-cells. However during their development CART is also present in beta-cells (21). The numbers of CART-IR endocrine cells are highly upregulated in rats with experimentally induced diabetes type 2 (1) or hypertension (14). Moreover it has also been documented that in humans, mice and rats CART serves to regulate the level of glucose by the increase of insulin 
and decrease of glucagon secretions (1). Additionally, some protective properties of CART in relation to rat beta cells has been demonstrated (19). What is the meaning of the finding that the endocrine pancreas of European bison is lacking CART-expressing endocrine cells is a matter of speculation at present, but suggests that some wild-life animal species may have different pathogenesis of CART-related type 2 diabetes.

In conclusion, we have demonstrated for the first time the region-specific CART expression patterns of the European bison pancreas. The obtained results suggest that in this animal species CART may influence both the endocrine and exocrine functions. The lack of CART-IR endocrine cells in the European bison gives rise new questions concerning the physiological and pathological role(s) of this neuropeptide in animals living in the wilderness.

\section{References}

1. Abels M., Riva M., Bennet H., Ahlqvist E., Dyachok O., Nagaraj V., Shcherbina L., Fred R. G., Poon W., Sörhede-Winzell M., Fadista J., Lindqvist A., Kask L., Sathanoori R., Dekker-Nitert M., Kuhar M. J., Ahrén B., Wollheim C. B., Hansson O., Tengholm A., Fex M., Renström E., Groop L., Lyssenko $V$., Wierup $N$.: CART is overexpressed in human type 2 diabetic islets and inhibits glucagon secretion and increases insulin secretion. Diabetologia 2016, 59, 1928-1937.

2. Arciszewski M. B., Barabasz S., Skobowiat C., Maksymowicz W., Majewski M.: Immunodetection of Cocaine- and Amphetamine-Regulated Transcript in the Rumen, reticulum, omasum and abomasum of the sheep. Anat. Histol. Embryol. 2009, 38, 62-67.

3. Arciszewski M. B., Calka J., Majewski M.: Cocaine- and amphetamineregulated transcript (CART) is expressed in the ovine pancreas. Ann. Anat. 2008, 190, 292-299.

4. Brooke-Smith M. E., Carati C. J., Bhandari M., Toouli J., Saccone G. T.: Galanin in the regulation of pancreatic vascular perfusion. Pancreas 2008 , 36, 267-273.

5. Cowles R. A., Segura B. J., Mulholland M. W.: Stimulation of rat pancreatic exocrine secretion by cocaine- and amphetamine-regulated transcript peptide. Regul. Pept. 2001, 99, 61-68.

6. Dolenšek J., Rupnik M. S., Stožer A.: Structural similarities and differences between the human and the mouse pancreas. Islets 2015, 7: e1024405
7. Douglass J., McKinzie A. A., Couceyro P.: PCR differential display identifies a rat brain mRNA that is transcriptionally regulated by cocaine and amphetamine. J. Neurosci. 1995, 15, 2471-2481.

8. Fujita T: : Histological studies on the neuro-insular complex in the pancreas of some mammals. Zeitschrift für Zellforschung 1959, 50, 94-109.

9. Gustavsen C. R., Pillay N., Heller R. S.: An immunohistochemical study of the endocrine pancreas of the African ice rat, Otomys sloggetti robertsi. Acta Histochem. 2008, 110, 294-301.

10. Holst J. J., Fahrenkrug J., Knuhtsen S., Jensen S. L., Poulsen S. S., Nielsen $O$. $V$ : Vasoactive intestinal polypeptide (VIP) in the pig pancreas: role of VIPergic nerves in control of fluid and bicarbonate secretion. Regul. Pept. 1984, 8, 245-259.

11. Ivanusic J. J., Goulding K. E., Kwok M. M., Jennings E. A.: Neurochemical classification and projection targets of CART peptide immunoreactive neurons in sensory and parasympathetic ganglia of the head. Neuropeptides 2012, 46, 55-60.

12. Janiuk I., Mtynek K.: Immunodetection of cocaine- and amphetamine-regulated transcript in bovine pancreas. Acta Histochem. 2015, 117, 545-550.

13. Kasacka I., Janiuk I., Lewandowska A., Bekisz A., Lebkowski W.: Distribution pattern of CART-containing neurons and cells in the human pancreas. Acta Histochem. 2012, 114, 695-659.

14. Kasacka I., Janiuk I., Piotrowska Z.: Evaluation of CART-, glucagon-, and insulin-immunoreactive cells in the pancreas of an experimental rat model of unilateral renal artery stenosis. Histol. Histopathol. 2015, 30, 445-452.

15. Kiba T: Relationships between the autonomic nervous system and the pancreas including regulation of regeneration and apoptosis: recent developments. Pancreas 2004, 29, 51-58.

16. Kirchgessner A. L., Gershon M. D.: Innervation of the pancreas by neurons in the gut. J. Neurosci. 1990, 10, 1626-1642.

17. Kirkwood K. S., Kim E. H., He X. D., Calaustro E. Q., Domush C., Yoshimi S. K., Grady E. F., Maa J., Bunnett N. W., Debas H. T.: Substance P inhibits pancreatic exocrine secretion via a neural mechanism. Am. J. Physiol. 1999, 277, G314-G320.

18. Kristensen P., Judge M. E., Thim L., Ribel U., Christjansen K. N., Wulff B. S., Clausen J. T., Jensen P. B., Madsen O. D., Vrang N., Larsen P. J., Hastrup S.: Hypothalamic CART is a new anorectic peptide regulated by leptin. Nature 1998, 393, 72-76.

19. Sathanoori R., Olde B., Erlinge D., Göransson O., Wierup N.: Cocaine- and amphetamine-regulated transcript (CART) protects beta cells against glucotoxicity and increases cell proliferation. J. Biol. Chem. 2013, 288, 3208-3218.

20. Wierup N., Gunnarsdóttir A., Ekblad E., Sundler F.: Characterization of CARTcontaining neurons and cells in the porcine pancreas, gastro-intestinal tract, adrenal and thyroid glands. BMC Neurosci. 2007, 8, 51.

21. Wierup N., Kuhar M. J., Nilsson B. O., Mulder H., Ekblad E., Sundler F.: Cocaine and amphetamine-regulated transcript (CART) is expressed in several islet cell types during rat development. J. Histochem. Cytochem. 2004, 52, 169-177.

Corresponding author: Marcin Arciszewski, Akademicka 12, 20-033 Lublin, Poland; e-mail: mb.arciszewski@wp.pl 